$10-8-2014$

\title{
Use of Grooved Microchannels to Improve the Performance of Membrane-Less Fuel Cells
}

\author{
J. D'Alessandro \\ Cleveland State University \\ Petru S. Fodor \\ Cleveland State University, p.fodor@csuohio.edu
}

Follow this and additional works at: https://engagedscholarship.csuohio.edu/sciphysics_facpub

Part of the Physics Commons

How does access to this work benefit you? Let us know!

Publisher's Statement

This is the accepted version of the following article: J. D'Alessandro and P. S. Fodor, "Use of Grooved Microchannels to Improve the Performance of Membrane-Less Fuel Cells," Fuel Cells 14 (6), 818-826 (2014). , which has been published in final form at http://onlinelibrary.wiley.com/ doi/10.1002/fuce.201400047/full

\section{Repository Citation}

D'Alessandro, J. and Fodor, Petru S., "Use of Grooved Microchannels to Improve the Performance of Membrane-Less Fuel Cells" (2014). Physics Faculty Publications. 207.

https://engagedscholarship.csuohio.edu/sciphysics_facpub/207

This Article is brought to you for free and open access by the Physics Department at EngagedScholarship@CSU. It has been accepted for inclusion in Physics Faculty Publications by an authorized administrator of EngagedScholarship@CSU. For more information, please contact library.es@csuohio.edu. 


\title{
Use of Grooved Microchannels to Improve the Performance of Membrane- Less Fuel Cells
}

\author{
J. D'Alessandro, P. S. Fodo
}

\begin{abstract}
In this work, the fluid dynamics within a membrane-less microchannel fuel cell is analyzed computationally. The membrane-less design is a result of the laminar nature of the fluid flow at small Reynolds numbers, restricting the fuel and oxidant to the vicinity of the corresponding electrodes, without the need of a proton exchange membrane (PEM). However, the performance of such cells is limited by the slow diffusive mass transport near the electrodes, with a large fraction of the reactants leaving the channel without coming in contact with the catalytic surfaces, and thus not being used. We mitigate this problem through the introduction of
\end{abstract}

\section{Introduction}

In recent years, the increasing reliance on portable electronic devices, such as cell phones, laptops, and tablets, has created a surge in social media and information technology. Not limited to this, the application of such devices has also spread to include medical diagnosis, environmental monitoring, and military technology. As the performance of portable electronics devices, in terms of speed, memory, and supported sensor platforms, continues to increase, so do their power requirements. Together with the requirement for more compact form factors this requires the development of new energy storage technologies with increased energy density. Currently, the majority of such devices employ batteries, such as the lithiumion type, to meet these energy needs. However, rechargeable batteries suffer from drawbacks such as performance degradation due to aging and long recharging times.

Fortunately advances in fabrication technologies have enabled both the miniaturization of conventional devices, as well as the exploration of novel modalities for energy storage/ conversion [1]. Among these, one class of devices with promising potential for improved performance when scaled down is miniature fuel cells. The advantages of this technology include: higher achievable power densitv due to the increased channel surface modification consisting of angled grooves designed to create convective flows that direct the reactants toward the active surfaces. The grooved structures are optimized for maximum fuel utilization. Operation of this type of cells at Péclet numbers close to 2,500 leads to a performance doubling compared with unmodified cells. Moreover, this increase in efficiency is accompanied by a more uniform distribution of the current across the electrodes, reducing the possibility of hot spots being developed.

Keywords: Computational Fluid Dynamics, Efficiency, Formic Acid Fuel Cell, Fuel Cell System, Mass Transport, Modeling, Numerical Simulation

surface-to-volume ratio leading to increased efficiency of electrochemical surface-based reactions; broad operating temperature ranges; and the elimination of recharging times as the energy can be stored in chemical packs outside of the cell $[2,3]$. Additionally, exploiting new design principles such as flow management in microfluidic fuel cells [4 9] and the development of 3D extended anode catalyst structures based on triple phase boundary localization of electrochemical reactions [10 12] has enabled recent prototypes to seek the elimination of the need for a proton exchange membrane (PEM). In conventional designs, the PEM constitutes the physical barrier preventing the mixing of fuel and oxidizer streams and their consequent fouling, while providing a transfer path for the protons between the catalysts covered electrodes at which the chemical reactions occur. However, the PEM is also the main component limiting the reliability of the fuel cell [13]. A major by-product of the chemical reactions taking place at the electrodes is water, and its management at the PEM has proved challenging. While the presence of water is necessary to promote the transfer of protons across the PEM, the membrane 
can become oversaturated, leading to an increase pressure drop across the membrane and decreased cell performance [14]. The reverse can also occur, leading to membrane fouling or dry out, due to increased operating temperatures and osmotic drag.

One of the widely used methodologies to design membrane-less microfluidic fuel cells relies on the laminar nature of the fluid flow in microscale devices, which are typically characterized by a low Reynolds number: $R e=\rho v L_{\mathrm{h}} / \eta$ (where $L_{\mathrm{h}}=4 \mathrm{area}_{\text {channel }} /$ perimeter $_{\text {channel }}$ is the hydraulic diameter of the channel, $\rho$ is the fluid density; $v$ is the speed; and $\eta$ is the fluid viscosity). In the simplest configuration, the fuel (Ex. formic acid, methanol) and oxidizer (oxygen saturated acidic solution) streams are fed through the separate arms of a T- or Yshaped channel (Figure 1) with the catalyst covered electrodes placed on the opposite channel walls. Because at low Reynolds numbers, the fluid motion is dominated by the inertial forces rather than the viscous ones, turbulent flows are absent, and the two redox streams (i.e., fuel and oxidizer) flow side-byside in the channel without mixing. Thus, the characteristic laminar flow defines a virtual interface between the fluid streams that plays the same role as a physical PEM. Besides the elimination of the PEM and of the issues associated with its long-term degradation, the co-laminar flow design is also highly adaptable in terms of the fuels used [4]. In recent years, research on this type of cells has led to designs with current/ power densities at levels that could warrant commercialization $[15,16]$.
Unfortunately, extensive studies of membrane-less cells have also shown that they are characterized by relatively poor fuel utilization $[4,16]$. The reason for the relatively poor fuel utilization is the diffusion limited mass transport near the anode and cathode, which leads to the formation of a depletion layer close to the electrodes following the initial reaction of the fuel and oxidant streams with the catalytic surfaces $[17,18]$. A longer residence time achievable through low flow rates allows the diffusional transport to partially overcome these boundary layers. However, this comes at the expense of decreasing the current density achievable. Moreover, the increased residence time also allows increased diffusional transport across the fuel/oxidizer interface and thus reactant crossover, which is detrimental to the operation of the cell. Increasing the flow rates increases the current density of the device and also limits the reactant crossover. However, the reduced residence times of the reactants near the electrodes, results in poor fuel utilization, with a large fraction of the reactants leaving the channel unused.

Since the introduction of the first membrane-less fuel cell designs, a sizable body of research has been dedicated to optimizing both the geometrical parameters, such as the aspect ratio and electrode configuration of the simple $\mathrm{T}$ - and $\mathrm{Y}$ shaped channels, as well as the inlet flow velocity in order to increase performance. However, the success in designing cells with both high current/power densities, high volume energy density, and efficient fuel utilization have met with limited success $[8,18,19]$. Encouraging results have been reported in designs that introduce modifications of the original geometry, through: the fabrication of porous electrodes to increase the contact area between reactants and electrodes and thus the fuel utilization [20,21]; the use of tapered channels [22] or H-shaped bridges [23] to limit the growth of the cross-over regions and allow the channels to be made sufficiently long for increased fuel residence time; or employing hydrodynamic focusing in which buffer supplied through a third inlet pushes the reactant streams against the electrodes [16]. In these studies notable improvements in the fuel utilization have been observed, while achieving performances in the current and power density comparable with those reported for state-of-the-art membrane-less designs using advanced 3D anode electrode designs [24]. Also, these microfluidic designs can be operated at high fuel concentrations and do not exhibit limitations related with the carbon dioxide gas bubbles accumulation near the cathode. However, some major drawbacks include

Fig. 1 (a) Basic design of the microchannel based fuel cell modeled; (b) surface oxidant concentra tion along the electrochemical cell. 
either the use of complex porous media or a greatly increased device size leading to low overall volume energy density.

In this study, we present an alternative modality to increase the efficiency of fuel consumption in membrane-less fuel cells, that relies on controlling the fluid flow close to the electrodes. A system of angled groove/ridges integrated on the bottom or/and top surface(s) of the cell's microchannel is used to induce transversal flows and promote increased mass transfer toward the electrodes. While the flow through the ridged channel is still laminar in nature, the addition of the ridges and the consequent introduction of a transversal component to the otherwise longitudinal flow, increases the amount of reactant that comes in contact with the catalytic electrode surfaces, overcoming the limitations imposed by the slow diffusion-based transport. This type of flow modification involving the generation of transversal flow components using fluid structure interactions, is similar with successful strategies used in some micro-scale mixers and reactors, in which the long timescale associated with diffusion-based transport imposes limitations on the efficiency of mixing and on the reaction rates [25 27]. In our work, the length and orientation of the ridges structures used is optimized to maximize the transversal flow close to the electrodes and thus the fuel consumption efficiency, while maintaining the integrity of the virtual membrane at the liquid liquid interface between the reactants. The analysis of the fuel/oxygen consumption rates indicates that this strategy leads to a notably increased performance of the cells in particular at larger flow rates where the strength of the induced transversal flows is increased. Thus, this strategy provides a solution to the challenge of achieving both high fuel utilization and high current density allowing the membraneless fuel cells to operate at higher reactant consumption rates at the flow rates that are associated with large current densities. Moreover, this modification can be easily integrated with previous approaches based on increasing the residence time of the reactants in the channel. For example, we show that the use of surface patterning together with hydrodynamic focusing $[8,16]$ leads to a doubling in the fuel/oxygen consumption compared with unmodified fuel cells. While the optimized fuel cell is slightly more geometrically complex than previously studied microchannel cells, its fabrication is still well within the means of multistep soft lithography methods [28].

\section{Computational Modeling}

All the simulations used in these work have been done using the Comsol Multiphysics Finite Element Analysis software and its Chemical Engineering module. The fuel is assumed to be $0.5 \mathrm{M}$ formic acid and the oxidant is dissolved oxygen, both in $0.1 \mathrm{M} \mathrm{H}_{2} \mathrm{SO}_{4}$ aqueous solution. The reactant consumption rates are modeled as a function of the flow rate through the channel. The data is analyzed in terms of the Péclet number $\left(P e=v L_{\mathrm{h}} / D\right.$, where $v$ is the fluid speed; $L_{\mathrm{h}}$ is the hydraulic diameter; and $D$ is the diffusion constant). The Péclet number is a measure of the relative importance of the advection and diffusion in chemical species transport. The range of Péclet numbers used is typically between 500 and 2,500 , and the flow is the low Reynolds number regime $R e \sim 1$. For all our simulations, the height $H$ of the channels is fixed at $100 \mu \mathrm{m}$ while their width $W$ is changed to obtain different aspect ratio channels. For each cell investigated, the maximum channel length is calculated based on the channel width and the Péclet number in order to limit the width of the diffusion layer between the fuel and oxidant streams. This is necessary to ensure that the fuel and oxidizer are confined to their corresponding electrodes along the entire length of the channel, in order to prevent reactant fouling.

\section{Fluid Dynamics}

In order to simulate the fuel and oxidant transport and consumption through the cell, first the flow fields in each structure are modeled by numerically solving the Navier Stokes equations of motion for an incompressible Newtonian fluid:

$\rho\left[\frac{\partial \mathbf{u}}{\partial t}+(\mathbf{u} \nabla) \mathbf{u}\right]=\nabla p+\eta \nabla^{2} \mathbf{u}$

$\nabla \mathbf{u}=0$

Here, $\mathbf{u}$ is the velocity vector, $\rho$ is the fluid density, $\eta$ is the fluid viscosity, $t$ is the time, and $p$ is the pressure. The equations are solved for a steady state flow with no slip boundary conditions at all surfaces and zero pressure at the outlet. The mesh used is a free tetrahedral one with the maximum element size less than $0.07 L_{\mathrm{h}}$ (where $L_{\mathrm{h}}$ is the hydraulic diameter of the channel). In all the geometries used, the length of the inlets is made long enough so that the fluid flow assumes a fully developed Poiseuille flow profile, by the time it reaches the main channel.

\section{Species Transport and Chemical Reactions}

Subsequent to obtaining the solution for the velocity field, for each of the species involved the diffusion convection equation is solved:

$\frac{\partial c_{i}}{\partial t}=D_{i} \nabla^{2} c_{i} \quad \mathbf{u} \nabla c_{i}$

In the above equation, the index $i$ indicates the fuel or oxidizer, and $c_{i}$ and $D_{i}$ are the corresponding concentrations and diffusions constant. For the fuel and oxygen diffusion coefficients we use $5 \times 10^{-10}$ and $2 \times 10^{-9} \mathrm{~m}^{2} \mathrm{~s}^{-1}$, respectively [8]. The concentrations of each species throughout the volume of the channel are obtained using a free tetrahedral mesh with a maximum size of $0.05 \mathrm{~L}_{\mathrm{h}}$. The mesh used is finer than that for the fluid dynamics to avoid numerical errors sometimes associated with modeling the diffusion convection equation.

The chemical reactions at the catalytic surfaces and the consumption of the fuel and oxidizer are modeled using negative fluxes at these surfaces determined by the corresponding reaction rate $k$ and concentration $c$. The rate of oxygen reduction at the cathode was calculated using the Butler Volmer equation [8]: 
$k_{\mathrm{O}}=\frac{i_{0}}{z F}\left(\mathrm{e}^{-\left(\alpha n F \eta_{\mathrm{c}} / R T\right)} \quad \mathrm{e}^{\left((1-\alpha) n F \eta_{\mathrm{c}} / R T\right)}\right)$

where $k_{\mathrm{O}}$ is the surface reaction rate for oxygen reduction, $i_{\mathrm{O}}$ is the exchange current density, $z$ is the valence, $F$ is the Faraday constant, $T$ is the temperature, $\alpha=0.5$ is the transfer coefficient rate, $n=1$ is the electron transfer number, $\eta_{C}=0.6 \mathrm{~V}$ is the overpotential at cathode, and $R$ is the universal gas constant. Given that the oxygen reduction rate is the limiting step $[5,8]$, the reaction rate for the fuel is adjusted to maintain global charge neutrality, and the discussion below focuses on analyzing the performance of the fuel cells in terms of oxygen utilization.

Similar computational fluid dynamics models have been previously used to model flows and chemical species distribution in channel based micromixers [26,29]. In regard to the addition of the surface reactions modeling the electrochemical reactions occurring at the electrodes, the model used was validated against the experimental results reported by Jayashree et al. [8] who used the same type of fuel, i.e., formic acid. For flow rates of $0.8 \mathrm{ml} \mathrm{min}^{-1}$, we found a reactant utilization rate of $7.9 \%$, which is well within the reported experimental value of $7.5 \pm 4 \%$ [8].

\section{Discussion}

Typical results showing the velocity field and the oxidant/ fuel concentrations in T shaped straight microfluidic channels are shown in Figure 2. For all the unmodified straight channel

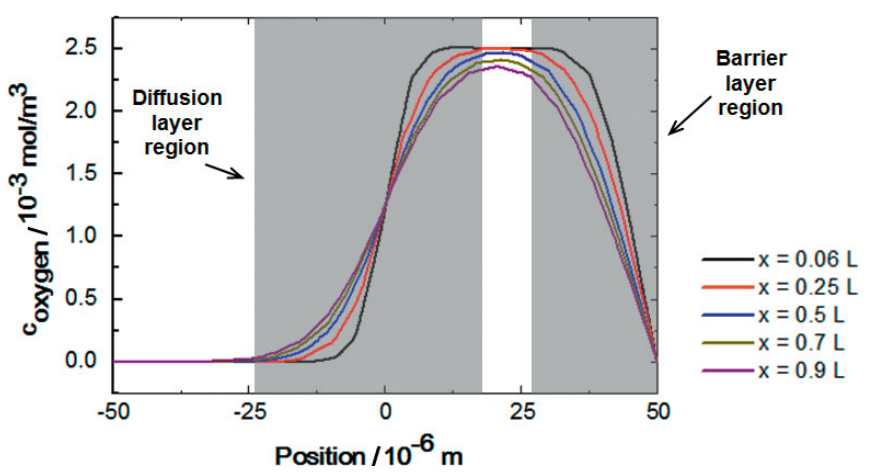

Fig. 3 Oxygen concentration line cuts through the middle of the cross sections at different positions along the channel $(P e=500)$. As one moves further downstream both the width of the diffusion layer (separat ing the fuel and oxidant streams) and the width of the barrier layer next to the electrodes increase.

cells investigated, the velocity field has the expected fully developed parabolic profile throughout the length of the main channel. On the other hand, as a result of the combined effect of the reactant advection/diffusion and reaction kinetics at the electrodes the oxidant/fuel concentration profiles are more complex. As described above, understanding the evolution of the oxidant concentration throughout the channel is particularly interesting as it provides a direct measure for the efficiency of the cell.

In the cross-sectional maps of the oxygen concentration, two distinctive features relevant to the performance of the cell are observed. Firstly, at the interface between the fuel and oxidant streams a diffusion layer forms as the chemical species are carried toward the outlet. The diffusion layer assumes an hourglass profile being wider near the top and bottom surfaces of the channel, where the flow rate is at minimum, and thinnest at the center of the channel where the flow rate is at a maximum. As shown in Figure 3, the width of the diffusion layer expands downstream in correlation with the increased residence time of the fuel/oxygen in the microchannel. The observed width of the diffusion layer close to the walls agrees well with the experimentally determined power law [30], $\Delta y \sim(D x)^{1 / 3}$, where $D$ is the diffusion constant and $x$ is the position along the channel. The downstream expansion of the diffusion layer is a limiting factor on the length of the channels that can be used as the reactant crossover to the incorrect electrode has an adverse effect on the functioning of the cell. Based on the above power law dependence of the width of the diffusion
Fig. 2 (a) Cross sections of the oxidant concentration at different positions along the channel (Pécle number $P e=500$ ); (b) close ups on the velocity field, the oxidant concentration, and the fuel concen tration, respectively. 
layer, for all the simulations the allowed length $L$ of the channels is determined as: $L=P e(0.2 W)^{3} /\left(H L_{\mathrm{h}}\right)$, so to avoid reactant crossover.

The second significant characteristic in the oxygen concentration evolution is the formation of a depletion boundary layer along the cathode. As oxygen reacts at the cathode catalytic surface, close to the electrode the fluid becomes depleted of free oxygen. The growth rate of the depletion layer toward the outlet of the channel is similar with that of the diffusion layer indicating that due to the characteristic laminar flow of the cell, the reactant transfer toward the electrodes is mediated primarily by diffusion. This has a dramatic effect on the functioning of the cell, as the diffusional transport is relatively slow. The effect of the inefficient reactant transfer toward the electrodes can be readily seen if the current density through the cathode electrode is mapped based on the surface reaction rates. As shown in Figure 4, the current density is at maximum at the inlet of the fuel cell and then diminishes rapidly toward the outlet, indicating that most of the reaction takes place before the depletion layer is formed. This negatively impacts efficiency of the cells, and also raises technical concerns in regard with the non-uniform current distribution and possible hot spots on the electrodes.

One strategy to mitigate somewhat the efficiency issue is to design cells with high aspect ratio $=$ width $(W) /$ height $(H)$ (Figure 5) [19,23,31]. As the aspect ratio of the cell is increased, while the flow and reactant concentration profiles share the same general features, the larger width allows for the channels to be made longer before the reactant crossover associated with the expansion of the diffusion layer affects their functionality. The increased residence time of the fuel/ oxygen in the cell associated with the increased channel length, allows for more oxygen to diffuse across the barrier layer and sustain the reaction at the cathode. Nevertheless, the achieved efficiencies (i.e., the ratio between the reactant flux at the outlet and the inlet, respectively) are modest $\sim 15 \%$ (Figure $5 b)$ since the reactant transport remains diffusion based and the length of the cell is still limited by the expansion of the interface layer between the fuel and oxidizer. Also, the

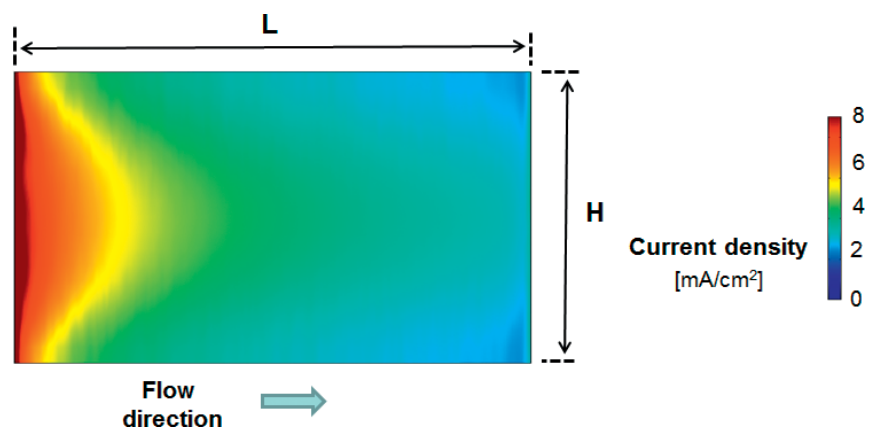

Fig. 4 Current density map for the cathode electrode for $P e=500$.
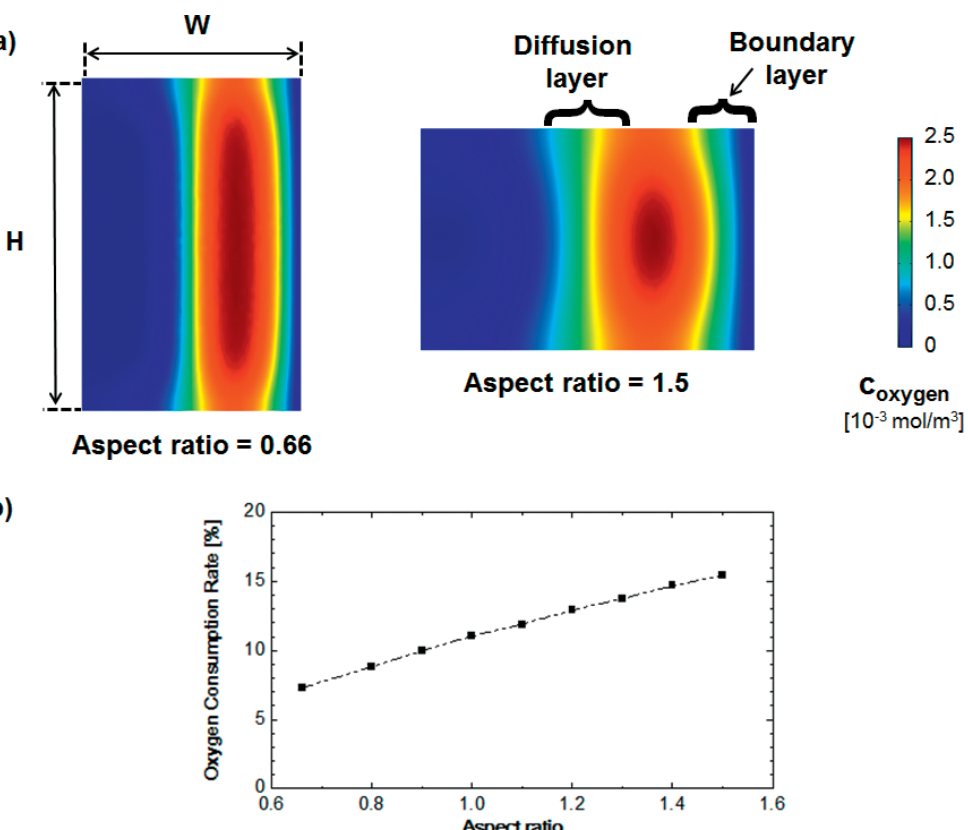

Fig. 5 (a) Cross sections of the oxygen concentration profiles at the outlet of chan nels with different aspect ratios at $P e=500$ (note: the aspect ratio is defined as the (dependence of the oxygen consumption ratio (note: the consumption rates are calcu ated by integrating the reactant flux across the entrance in the main channel and cross the outlet, respectively).

decrease in the volume energy density of the cell associated with the larger widths/lengths outpaces the gains in efficiency.

It is thus apparent that improvement of the microchannel based fuel cell efficiency is dependent on overcoming the slow diffusional reactant transport across the depletion boundary layer. The challenge in achieving this is similar with that encountered in designing microreactors where the laminar nature of the fluid flow and the slow diffusional transport hinder the volume mixing of chemical reactants. One of the strategies employed in promoting reactant transfer in diffusion limited microchannel reactors, that is transferable to the case of membrane-less fuel cells, is channel surface modification $[25,26,29]$. Essentially, this method relies on the addition of a system of ridges/grooves to the bottom and/or top surface of the microfluidic channel (Figure 6). In these geometries, the resistance to the axial motion of the fluid presented by the slanted ridges, transfers momentum from the longitudinal flow into transversal components. In particular for the case of the modified fuel cells presented here, velocity field calculations show the formation of counter-rotating transversal flows on the sides of the channel. As shown in Figure 6c, for the particular ridge orientation employed, i.e., angled toward the outlet, the transversal flow patterns drive the reactants form the center of the channel toward the middle of the catalytic surfaces and then across them, maximizing the contact between the reactants and the electrodes. Thus, the chemical species advection induced by these surface modifications, effectively provide the means for mass transfer from the reac- 


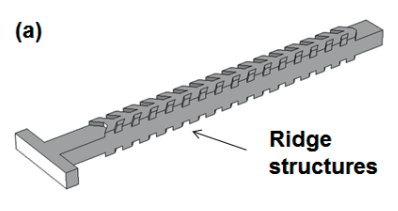

(b)

(c)

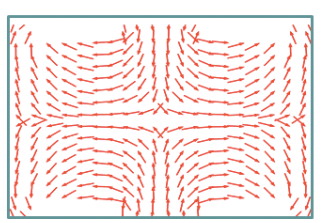

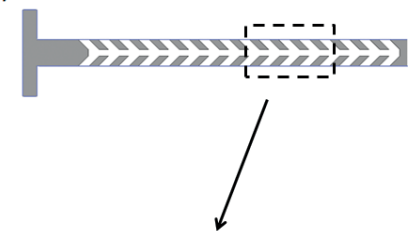

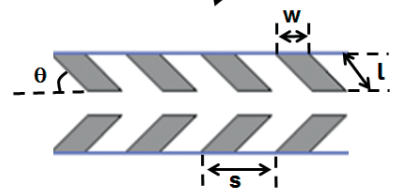

Fig. 6 (a) Modified microchannel designs using ridges on the top and bottom of the channel; (b) planar projection of the ridge structure with the basic parameters defining its geometry; (c) arrow plot of the transver sal flows generated by the system of slanted ridge/grooves $(P e=750)$.

tant rich center of the channel to the depleted regions near the electrodes and mitigate the limits on the efficiency of the fuel cell imposed by the slow diffusional transport. The outcome of the increased reactant transfer toward the catalytic surfaces is immediately apparent in the oxidant concentration profiles along the channels as shown in Figure 7 for fuel cells with aspect ratio 1.5 . For the designs with surface modifications, the depletion region is narrower and the average and maximum oxidant concentration at the outlet are reduced indicating better fuel utilization as expected based on the induced convective contribution to the reactant transport toward the electrodes. This effects are more pronounced in the cells with structures on both the top and bottom of the channel, in correlation with the better developed transversal flows in these cases.

Given that the performance of the surface modified cells is dependent on the induced transversal flows it becomes important to maximize them. The strength of these flow patterns is determined by the longitudinal pressure gradient between the

(a)
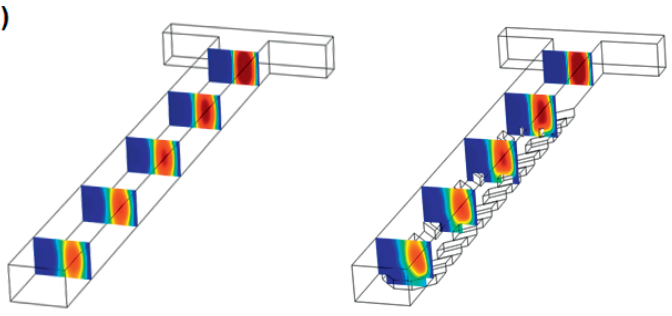

(b)

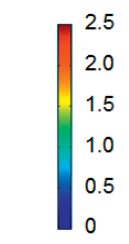

Coxygen $\left[10^{-3} \mathrm{~mol} / \mathrm{m}^{3}\right]$

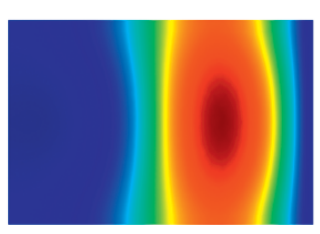

No ridges

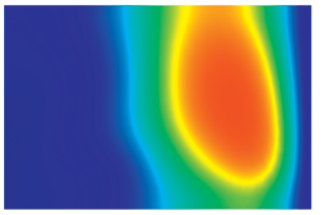

Ridges on the bottom surface
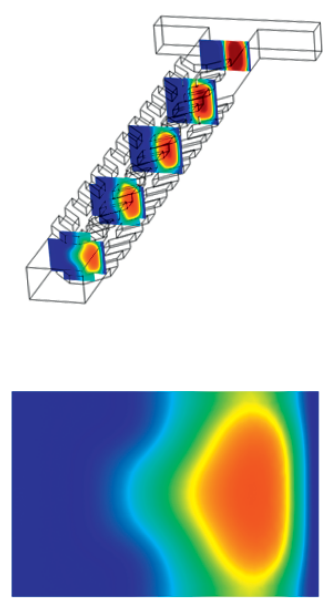

Ridges on the top and bottom surface

Fig. 7 (a) Evolution of the oxygen concentration along the channel for cells with various geome tries; (b) oxygen concentration profiles at the outlet (for all the channels shown $W=150 \mu \mathrm{m}$, $H=100 \mu \mathrm{m}$, and $P e=750)$.

inlet and outlet and by the geometrical parameters defining the ridge/groove structures that include: the angle $\theta$ with respect to the channel axis; the groove's depth $h$, width $w$, and length $l$; and the spatial periodicity $s$. Thus, the search space for the optimal geometrical parameters is quite large. Fortunately, a large body of experimental, analytical, and computational work, on passive straight channel micromixers, has been dedicated to their geometry optimization [32 35]. Some of the determined geometrical dimensions that ensure maximization of the transversal flow components and vorticity include: ridge orientation $\theta=45^{\circ}$ with respect to the fluid flow direction [25]; ridge depth as a function of the main channel height $h=0.25 H[32,33]$; ratio of groove width $w$ to pitch $s$ $\sim 0.5$ to $0.7[26,36]$. Thus for the fuel cells modeled (aspect ratio $=1.5, W=150 \mu \mathrm{m} ; H=100 \mu \mathrm{m}$ ) in correlation with the previous optimization work the dimensions chosen were $h=50 \mu \mathrm{m}, w=65 \mu \mathrm{m}$, and $s=115 \mu \mathrm{m}$, respectively. It has to be noted though, that while the optimization work on inducing transversal flows in micromixers is a valuable resource in guiding the geometrical parameter choice for our fuel cell design, its scope is mainly concerned with maximizing volume mixing rather than directing mass transport toward specific surfaces. This is an important difference, since while the transversal component added to the flow has the beneficial effect of mitigating the reaction depletion near the electrodes, it can also disturb the integrity of the virtual membrane at the liquid liquid interface between the fuel and oxidant streams. Disruption of the diffusion layer, which constitutes the physical barrier separating the reactants can be detrimental to the functioning of the cell, as a fraction of the chemical species can be advected to the incorrect side of the cell (i.e., oxidant to the anode and fuel to the cathode, respectively) leading to increased reactant crossover. This effect can be mitigated by the careful choice of the length parameter $l$ of the ridges, which is one of the primary factors determining the extent by which the transversal flows extend into the diffusion layer region. To quantify the influence of the ridge length on the performance of the cells, channels with ridges with lengths up to $W \sqrt{ } 2 / 2$, i.e., spanning the entire width of the channels, were investigated (Figure 8). As the ridge length is increased, the efficiency of the cells monotonously increases in relation with the larger contact area between the fluid stream and the flow modifying features and consequently the increased fluid-structure interaction. As expected, monitoring the oxygen concentration at the outlet shows that the increased efficiency is accompanied by a slight undesirable increase in the oxygen concentration near the anode. However, this increase is minimal when compared with the residual oxygen concentration in the unmodified channels (Figure 8b). More- 
(a)

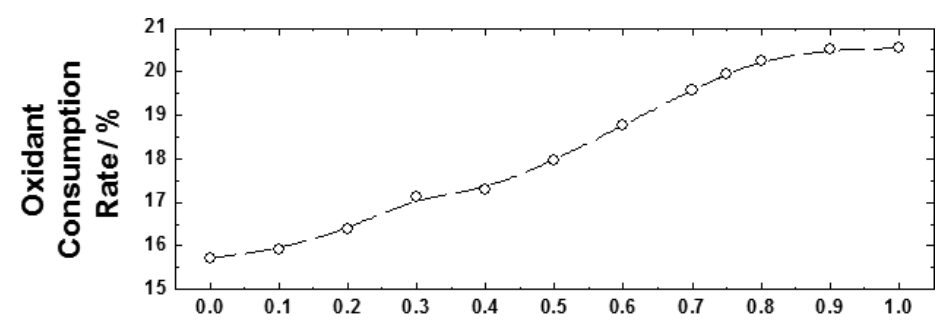

(b)

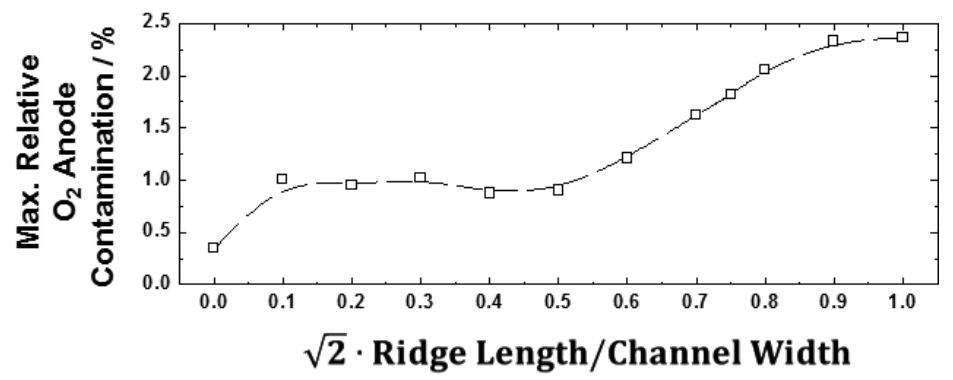

Fig. 8 Ridge length dependence of the (a) oxidant consumption rate and (b) oxygen contamination at the anode, respectively (note: the oxygen contamination is normalized by the maximum oxygen concentration in the cell).

over, since as shown in Figure 8a, the efficiency of the cells plateaus as the ridges' length is increased, in all the following studies the $l$ parameter is limited to 0.75 of the maximum possible value. This allows the cells to operate close to the maximum achievable efficiency, while leaving the central part of the channel free of surface modification, and thus ensuring that the transversal flows are primarily localized near the electrodes and their effect is minimized in the diffusion region near the center of the channel.

The superiority of the ridged channels is also revealed by the current density profiles across the cathode (Figure 9). While for the unmodified channels the electrochemical reactions are quickly suppressed after the formation of the depletion layers, current density maps for the ridged channels illustrate the ability of the transversal flows to continually replenish the reactant near the electrodes and sustain the electrochemical reactions. The relationship between the extent of the transversal mass transport induced by the surface modifi-
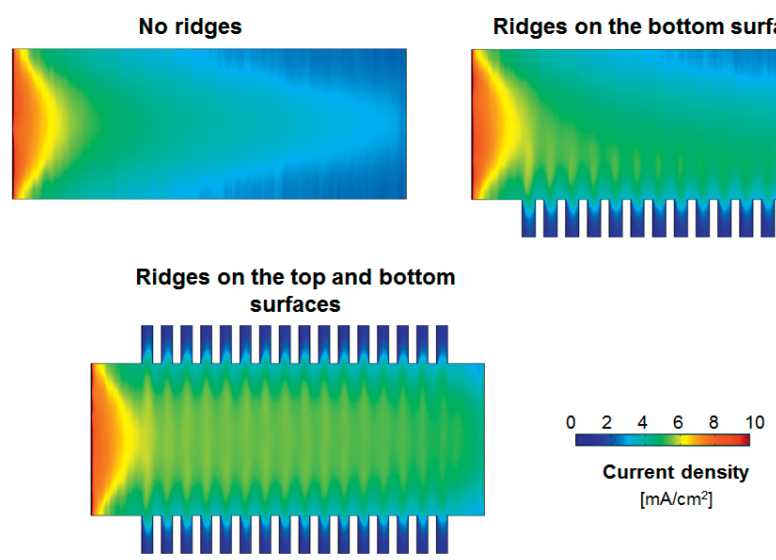

Fig. 9 Current density for channels with various geometries. For all the channels shown $W=150 \mu \mathrm{m}, H=100 \mu \mathrm{m}$, and $P e=750$ ). cations and the local reaction efficiency is particularly evident when comparing current density maps in channels with ridges only on the bottom surface, with channels containing ridges on both boundaries. In the former, the reaction rate is clearly maximized only near the modified surface. Modifying both surfaces as in the later design ensures uniform utilization of the entire electrode surface.

Based on the strong correlation between the increased reactant utilization rates and the presence of the transversal flows, one expects that increasing the strength of the cross-sectional transport will lead to higher efficiencies. One straightforward way to increase the strength of the cross-sectional flows in our design is to operate the fuel cell at higher Péclet numbers, i.e., higher inlet speeds. In essence, increased flow rates correlate with stronger fluid ridge structure interaction and consequently more momentum transfer toward cross-sectional flows and increased reactant transport across the depletion layer. Figure 10 shows results for the utilization rates extracted from simulations of the fluid flow and reaction kinetics at increasing Péclet numbers. As expected the efficiency of the cells with surface modifications, steadily improves as the flow rate is increased. Similar performance improvements have been recently observed experimentally in a co-laminar boronhydride cell with surface modifications [37]. However, due to the electrolyte used and the higher potential associated with the reactants employed in the study, a quantitative assessment of the effect of the ridge/groove structures on the performance of the cell could not be easily made [9]. The current study indicates that the performance can correlate strongly with the presence of surface modifications, as a similarly dramatic increase of the efficiency at high Péclet numbers is not observed in channels without surface modifications (Figure 10). This is because in unmodified designs there is no mechanism to translate the high flow rates into increased cross-sectional transport.

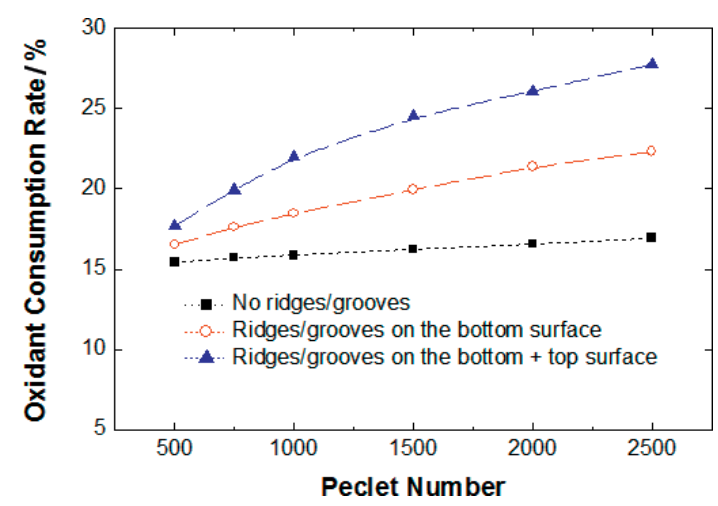

Fig. 10 Comparison of the cell performance as a function of the Péclet number for the unmodified and surface patterned channels. 
(a)

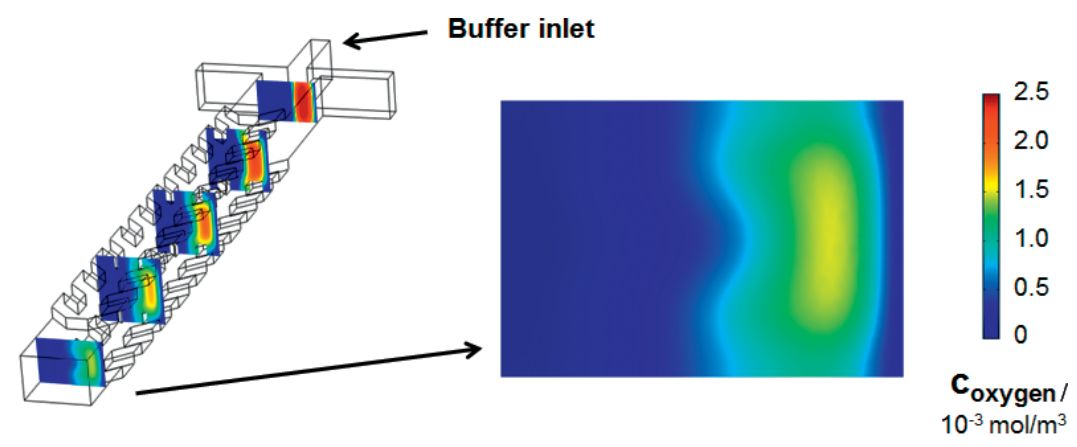

(b)

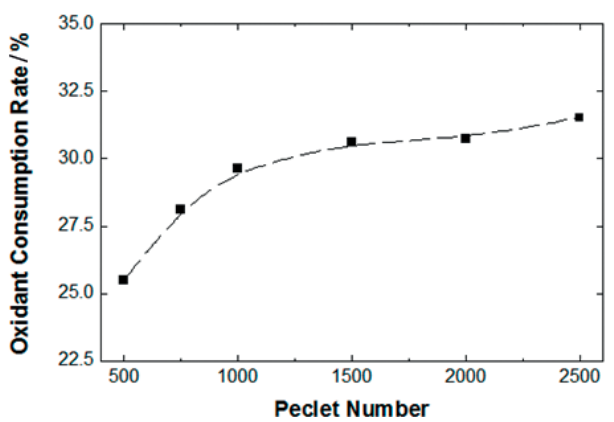

Fig. 11 (a) Evolution of the oxygen concentration along the channel for a cell with surface patterning including a buffer inlet; (b) cell performance as a function of the Péclet number.

One of the advantages of the proposed solution to the reactant utilization problem in membrane-less fuel cells is its potential for seamless integration with other strategies previously used for improving their performance. As an example, the surface modifications proposed can be easily integrated in designs using hydrodynamic focusing, where a stream of buffer fed into the channel is used to push the reactants against the electrodes and diminish the extent of the depletion layer. An analysis of the performance of this design (Figure 11b) shows a very good performance in terms of reactant consumption rate. Operation of this type of cells at high Péclet numbers leads to almost a doubling in terms of reactant utilization efficiency relative to unmodified straight channel cells and a similar increase in the average current density. Moreover, the increased width of the interface layer between the fuel and oxidizer, associated with the presence of the buffer stream makes the diffusion layer less susceptible to disruption by the transversal flows that promote the transport of fuel and oxidizer toward the active surfaces. As shown in the oxygen concentration cross-section at the outlet (Figure 11a) for the ridge channel + buffer inlet, the disturbance in the interface layer between the oxygen and fuel streams and the reactant crossover are minimal.

\section{Summary}

Simulations of fluid flow, reactant transport, and surface reactions in microchannel fuel cells have been used to identify strategies for promoting efficient reactant consumption. Inspired from the use of surface modification strategies in microreactors to manipulate fluid flow, a new type of micro fuel cell using a slanted ridge/groove geometry has been investigated. The use of ridge structures promotes transversal flows close to the catalytic electrodes and thus mitigates the reactant depletion in the active region due to slow diffusion. The improvement in the efficiency of the cells is larger at high flow rates due to the maximization of the transversal components of the flow. Surface pattering of the channels are easily integrated with previously studied strategies to address these challenges, such as the addition of a buffer layer between the reactant streams. In this type of designs, we achieved a doubling of the fuel utilization when compared with the unmodified cells. Thus, the methods described demonstrate the potential to break the trade-off between achieving high fuel utilization and high current density, when operating in the high Péclet number regime.

\section{Acknowledgment}

Part of this work has been supported through the Cleveland State University Provost Summer Research for Undergraduates fund.

\section{References}

[1] S. Pennathur, J. C. T. Eijkel, A. van den Berg, Lab Chip 2007, 7, 1234 .

[2] Y. Vilenchik, E. Peled, D. Andelman, Phys. World 2010, 23, 20.

[3] A. Kundu, J. H. Jang, J. H. Gil, C. R. Jung, H. R. Lee, S.-H. Kim, B. Ku, Y. S. Oh, J. Power Sources 2007, 170, 67. 
[4] E. Kjeang, N. Djilali, D. Sinton, J. Power Sources 2009, $186,353$.

[5] E. Choban, J. S. Spendelow, L. Gancs, A. Wieckowski, P. J. A. Kenis, Electrochim. Acta 2005, 50, 5390.

[6] R. Ferrigno, A. D. Stroock, T. D. Clark, M. Mayer, G. M. Whitesides, J. Am. Chem. Soc. 2002, 124, 12930.

[7] J. L. Cohen, D. A. Westly, A. Pechenik, H. D. Abruña, J. Power Sources 2005, 139, 96.

[8] R. S. Jayashree, S. K. Yoon, F. R. Brushett, P. O. LopezMontesinos, D. Natarajan, L. J. Markoski, P. J. A. Kenis, J. Power Sources 2010, 195, 3569.

[9] M. Goulet, E. Kjeang, J. Power Sources 2014, 260, 186.

[10] A. Lam, D. P. Wilkinson, J. Zhang, Electrochim. Acta 2008, 53, 6890 .

[11] A. Lam, B. Wetton, D. P. Wilkinson, J. Electrochem. Soc. 2011, 158, B29.

[12] M. S. Dara, K. Fatih, D. P. Wilkinson, J. Electrochem. Soc. 2014, 161, F105.

[13] F. A. de Bruijn, V. A. T. Dam, G. J. M. Janssen, Fuel Cells 2008, 8, 3 .

[14] X. Liu, H. Guo, F. Ye, C. F. Ma, Electrochim. Acta 2007, 52, 3607.

[15] J. Lee, K. G. Lim, G. T. R. Palmore, A. Triathi, Anal. Chem. 2007, 79, 7301.

[16] J. Xuan, M. K. H. Leung, D. Y. C. Leung, M. Ni, H. Wang, Int. J. Hydrogen Energy 2011, 36, 11075.

[17] E. R. Choban, P. Waszczuk, P. J. A. Kenis, Electrochem. Solid State Lett. 2005, 8, A348.

[18] A. Bazylak, D. Sinton, N. Djilali, J. Power Sources 2005, 143, 57.

[19] M. H. Chang, F. Chen, N. S. Fang, J. Power Sources 2006, $159,810$.

[20] E. Kjeang, R. Michel, D. A. Harrington, N. Djilali, D. Sinton, Am. Chem. Soc. 2008, 130, 4000.
[21] K. S. Salloum, J. D. Posner, J. Power Sources 2010, 195, 6941.

[22] A. E. Khabbazi, A. J. Richards, M. Hoorfar, J. Power Sources 2010, 195, 8141.

[23] H. B. Park, D. H. Ahmed, K. H. Lee, H. J. Sung, Electrochim. Acta 2009, 54, 4416.

[24] A. Lam, M. S. Dara, D. P. Wilkinson, K. Fatif, Electrochem. Commun. 2012, 17, 22.

[25] A. D. Stroock, S. K. Dertinger, A. Ajdari, I. Mezic, H. A. Stone, G. M. Whitesides, Science 2002, 295, 647.

[26] P. S. Fodor, M. Itomlenskis, M. Kaufman, Eur. Phys. J.: Appl. Phys. 2009, 47, 31301.

[27] S. K. Yoon, G. W. Fichtl, P. J. A. Kenis, Lab Chip 2006, 6, 1516.

[28] J. C. McDonald, D. C. Duffy, J. R. Anderson, D. T. Chiu, H. Wu, J. A. Shuller, G. M. Whitesides, Electrophoresis 2000, 21, 27.

[29] P. S. Fodor, M. Kaufman, Mod. Phys. Lett. B 2011, 25, 1111.

[30] R. F. Ismagilov, A. D. Stroock, P. J. A. Kenis, G. Whitesides, Appl. Phys. Lett. 2000, 76, 2376.

[31] D. H. Ahmed, H. B. Park, H. J. Sung, J. Power Sources 2008, 185, 143.

[32] P. Sundararajan, A. D. Stroock, Annu. Rev. Chem. Biomed. Eng. 2012, 3, 473.

[33] M. S. Williams, K. J. Longmuir, P. Yager, Lab Chip 2008, 8, 1121 .

[34] N. S. Lynn, D. S. Dandy, Lab Chip 2007, 7, 580.

[35] H. Z. Wang, P. Iovenitti, E. Harvey, S. Masood, Micromech. Microeng. 2003, 13, 801.

[36] A. Afzal, K. Y. Kim, Sens. Actua. B 2014, 192, 350.

[37] N. Da Mota, D. A. Finkelstein, J. D. Kirtland, C. A. Rodriguez, A. D. Stroock, H. D. Abruña, J. Am. Chem. Soc. 2012, 134, 6076.

Post-print standardized by MSL Academic Endeavors, the imprint of the Michael Schwartz Library at Cleveland State University, 2017. 